\title{
Correction: MYC-containing amplicons in acute myeloid leukemia: genomic structures, evolution, and transcriptional consequences
}

\author{
Alberto L'Abbate ${ }^{1} \cdot$ Doron Tolomeo $^{1} \cdot$ Ingrid Cifola $^{2} \cdot$ Marco Severgnini ${ }^{2}$ - Antonella Turchiano ${ }^{1}$. \\ Bartolomeo Augello ${ }^{3}$ - Gabriella Squeo ${ }^{3}$ - Pietro D'Addabbo $\mathbb{D}^{1}$ - Debora Traversa ${ }^{1} \cdot$ Giulia Daniele $^{1}$. \\ Angelo Lonoce ${ }^{1}$ - Mariella Pafundi ${ }^{1}$ - Massimo Carella ${ }^{3}$ - Orazio Palumbo $\mathbb{D}^{3} \cdot$ Anna Dolnik $^{4}$. \\ Dominique Muehlematter ${ }^{5}$. Jacqueline Schoumans ${ }^{5} \cdot$ Nadine Van Roy $^{6} \cdot$ Gianluca De Bellis $^{2} \cdot$ Giovanni Martinelli $^{7}$. \\ Giuseppe Merla $^{3} \cdot$ Lars Bullinger $^{4} \cdot$ Claudia Haferlach $^{8} \cdot$ Clelia Tiziana Storlazzi $^{1}$
}

Published online: 9 July 2018

(c) Macmillan Publishers Limited, part of Springer Nature 2018

Correction to: Leukemia; https://doi.org/10.1038/s41375018-0033-0; published online 22 Feb 2018

In the original version of this Article, the affiliation details for Giovanni Martinelli were incorrectly given as 'Department of Experimental, Diagnostic and Specialty Medicine, University of Bologna, 40138, Bologna, Italy' and it should have been given as 'Istituto Scientifico Romagnolo per lo Studio e la Cura dei Tumori (IRST) IRCCS, Meldola, Italy and not Department of
Experimental, Diagnostic and Specialty Medicine, University of Bologna, 40138, Bologna, Italy.'

Furthermore, the original version of this Article contained an error in the spelling of the authors Alberto L'Abbate and Pietro D'Addabbo, an acute accent was incorrectly used instead of an apostrophe for these authors names.

These errors have now been corrected in both the PDF and HTML versions of the Article.

Clelia Tiziana Storlazzi

cleliatiziana.storlazzi@uniba.it

1 Department of Biology, University of Bari "Aldo Moro",

Bari, Italy

2 Institute for Biomedical Technologies (ITB), CNR, Segrate, Italy

3 Division of Medical Genetics, IRCCS 'Casa Sollievo della Sofferenza', San Giovanni Rotondo, Italy

4 Department of Internal Medicine III, University Hospital of Ulm, Ulm, Germany

5 Centre Hospitalier Universitaire Vaudois (CHUV), Lausanne University Hospital, Lausanne, Switzerland

6 Center for Medical Genetics, Ghent University, Ghent, Belgium

7 Istituto Scientifico Romagnolo per lo Studio e la Cura dei Tumori (IRST), IRCCS, Meldola, Italy

8 Munich Leukemia Laboratory (MLL), Munich, Germany 